\title{
4D Scanning of Clothed Humans - Preliminary Results
}

\author{
Tino KÜHN, Yordan KYOSEV \\ TU Dresden, Institute of Textile Machinery and High Performance Material Technology, \\ Chair of Development and Assembly of Textile Products, Germany
}

https://doi.org/10.15221/21.25

\begin{abstract}
Modern hardware makes high speed 3D scanning (4D scanning) of moving clothed humans possible and opens new possibilities for optimization of the pattern in order to provide improved clothing comfort. At the same time the high speed scanning process generates a large amount of data which analysis over times series is not more as efficient as it is for single 3D frames. This contribution reports first steps in the area of evaluation of high speed scanned data sets of humans with not tight clothing. The newest export of the IBV scanner MOVE4D in the form of triangulated meshes of the complete scan (human and textiles) is critically analyzed. It was recognized that it provides an efficient initial mesh for additional analysis from one side and some effects like small folds and areas, where the cloth is sticking to the body cannot be recognized anymore. After that the method of the approximate distances between different raw data cloud points are analyzed for a raising arms motion of a clothed human. The optical analysis demonstrates good correlation with the reality and suitability of the method for fast analysis of the clothing behavior based on 4D scanned frames.
\end{abstract}

Keywords: 4D body scanning, data processing, cloud point distances, motion, clothed human

\section{Introduction}

The fit of the clothing is important for the wear comfort. The most current fitting evaluation tools visualize the distance between the human body and the clothing or the pressure over the human body in "distance or pressure maps", as a parameter for the objective evaluation of the fit. Most of these methods are based on static postures and do not provide the information about the dynamic interaction between the clothing and the human body. The modern high-speed scanning technique, as for instance the MOVE4D scanner of IBV [1] and the 3DMD scanners [2], provide new possibilities for the analysis of the workwear and any other functional or everyday clothing. As result of the motion scanning with high frequency, a large set of point cloud data are recorded with a volume between 30 and $80 \mathrm{MB}$ per frame. One main research task is the automatic evaluation of such series of 3D scans and the extraction of the relevant parameters for the current design applications.

\section{State-of-the art}

Scanning of moving humans with clothing is important for the evaluation of the clothing comfort during the motion and for evaluation of the air gap between the body and the clothing [3, 4]. Initially for such analysis single 3D frames were used, wherefrom a few postures are analyzed [5, 6]. The classical 3D scanners require longer scanning and processing time per frame and are not able to catch detailed dynamic information for the body during the motion. Another approach is the application of motion capturing systems [7], normally used in the health care, sport posture optimization and film character creation. Such system is able to track several points of the body during the motion, but does not contain information for the whole body part. This information have to be recreated using rigging of the body [8] and morphing of the human body mesh based on specialized algorithms.

A powerful method is the use of trained data based 3D models for the human body and a large set of pictures, as reported in [9]. The most complete system for such analysis is the ClothCap approach [10], developed to process the data of 4D scanner at 60 frames per second. It consists of several parts, which include segmentation of the single parts of the body, estimation of the naked body shape and pose and tracking the clothing deformation over the time [10]. This work presents first preliminary results on analysis of the 4D scanning data only, without human templates, for fast processing and analysis of the clothing. The analysis of the scan data set has not the power of multi-part approach of ClothCap [10] and is not able to separate the human body from the clothing, but can be used for quick checks of the clothing under motion and in this way to support the development and optimization of the clothing. 


\section{4D Scanner and data formats}

The 4D scanning is performed with the MOVE4D scanner [1], available in the scanning lab of the ITM, TU Dresden. The scanner consists of 12 scanning modules, which are able to record motion with special resolution up to $1 \mathrm{~mm}$ and up to 180 frames per second. After the processing of the data by the IBV software, various options for data processing and export are available:

- The raw point clouds are available as PLY format. These consists of $x, y, z$ coordinates of each voxel and its red, green and blue color values.

- Template fitted of water tight meshes of the human bodies, usefull only for close to body clothing, exported as OBJ format including the texture map. This requires A-Pose scan and rigging data (FBX)

- Non-holonomic triangulated mesh as OBJ format and texture for objects with free moving clothing. This new option is evaluated here.

In this work the main task was the analysis of the scanned clothing from the raw point data and from the triangulated mesh. The water tight mesh and the rigging are not subject of this investigation.

\section{Results}

\subsection{Triangulated mesh of non close to the body clothing}

A masculine human was scanned with office clothing of normal fit trousers with belt and shirt (Fig. 1). A series is selected by the motion of moving both hands from down to up, performed in approx. 2-3 seconds and scanned with 30 frames per second. The initial frame, and frames 40, 80 and 100 are presented in Fig 1. The visual analysis of the meshes shows realistic and close to the reality wrinkles. At some folds the mesh is not closed, but this can be solved by postprocessing and does not presents problem in the analysis of the data.

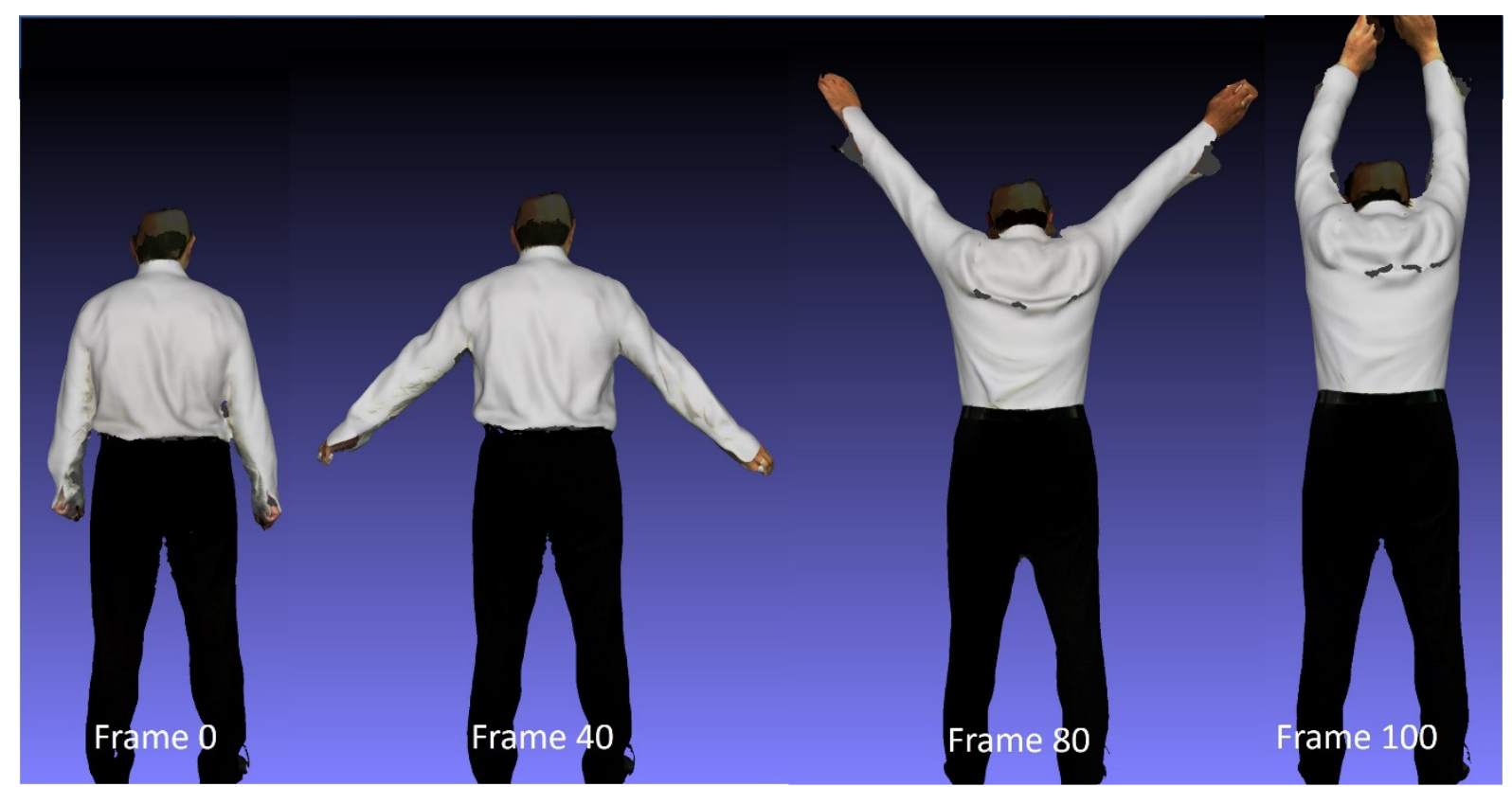

Fig. 1. Selected frames, saved as triangulated mesh.

Closer look and comparison between the triangulated mesh at frame 100 and the raw data (Fig 2) demonstrates that doe to the smoothing of the points to the triangle mesh, two kinds of information gets lost:

- A larger number of small folds and wrinkles are recognizable on the raw point data (Fig 2), which are not more recognizable in the triangulated mesh of the same frame. The reason for this is simple - the folds are in similar size as the mesh in this case. If they have to be kept, the mesh should become significantly finer, which will require larger memory.

- The area, where the shirt is contacting tight to the body is well optically recognizable on the raw data point, but not more in the triangulated mesh. 


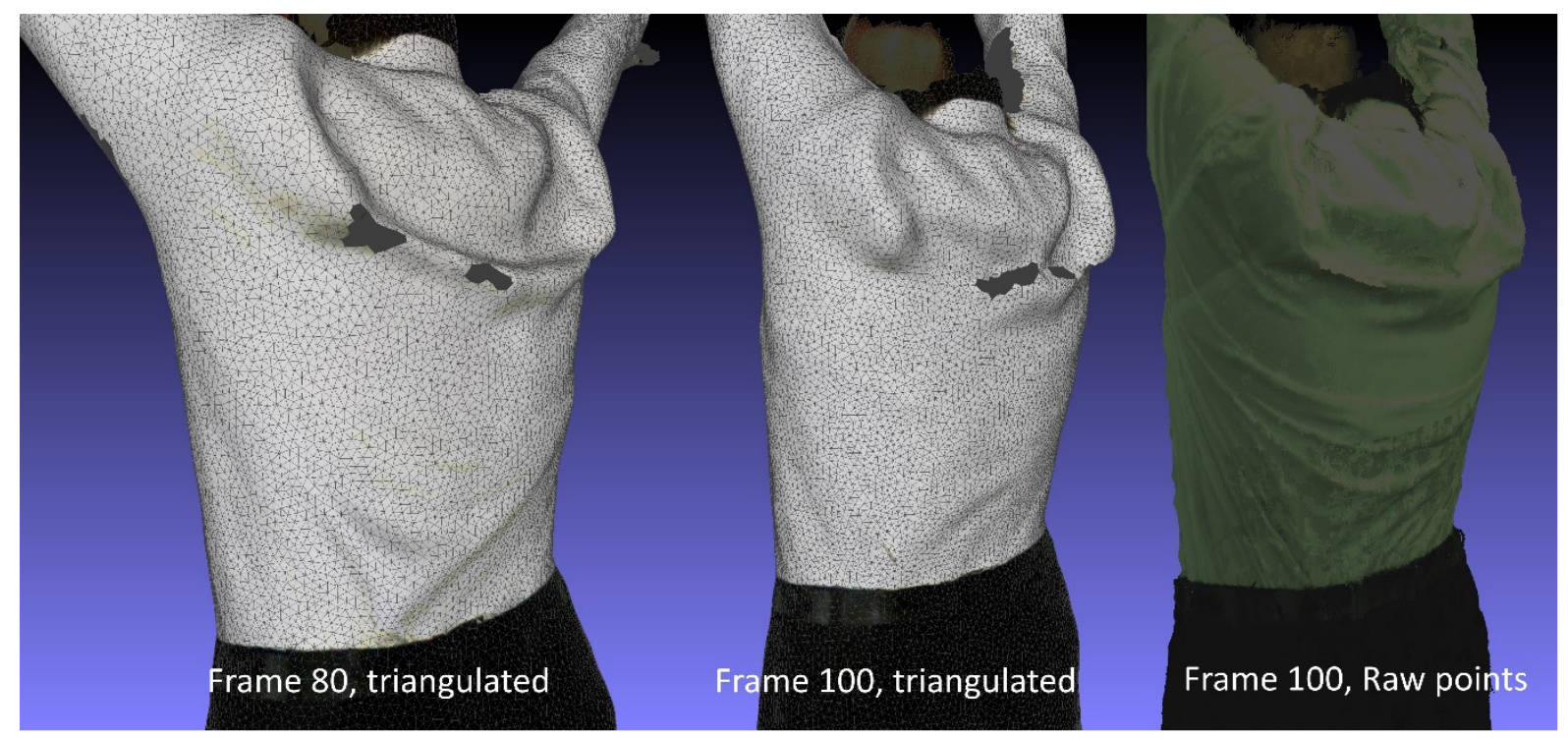

Fig. 2. Frames 80 and 100 triangulated and frame 100 as raw data points.

\subsection{Raw data point analysis}

The raw data point clouds contain more optical information, which is not filtered and could be used for direct analysis. In the current case the clout points were compared by using the CloudCompare software [11], which provide the functionality of computation of distances between two point clouds, based on the nearest neighbor algorithm. Using this algorithm, for each point $p_{i}\left(x_{i}, y_{i}, z_{i}\right)$ of the compared cloud, the nearest point $P_{i}\left(X_{i}, Y_{i}, Z_{i}\right)$ in the reference cloud is searched and their Euclidean distance is computed:

$$
L_{i}=\sqrt{\left(x_{i}-X_{i}\right)^{2}+\left(y_{i}-Y_{i}\right)^{2}+\left(z_{i}-Z_{i}\right)^{2}}
$$

This algorithm obviously does not provide the exact deformation or slippage of the clothing, it provides only the information about the motion of the mesh, but it has the potential to provide fast approximative analysis of the clothing motion between the frames. If in one area the textile clothing is sliding but in the same geometry another piece of textile remains, it can happens, that the algorithm detects zero distance - no slippage of the mesh, but the textile is moving. But as the algorithm has quick implementations in various software libraries [11,12] and the scanned mesh from the Move4D scanner is dense enough, it has potential to provide good approximation of the clothing motion.
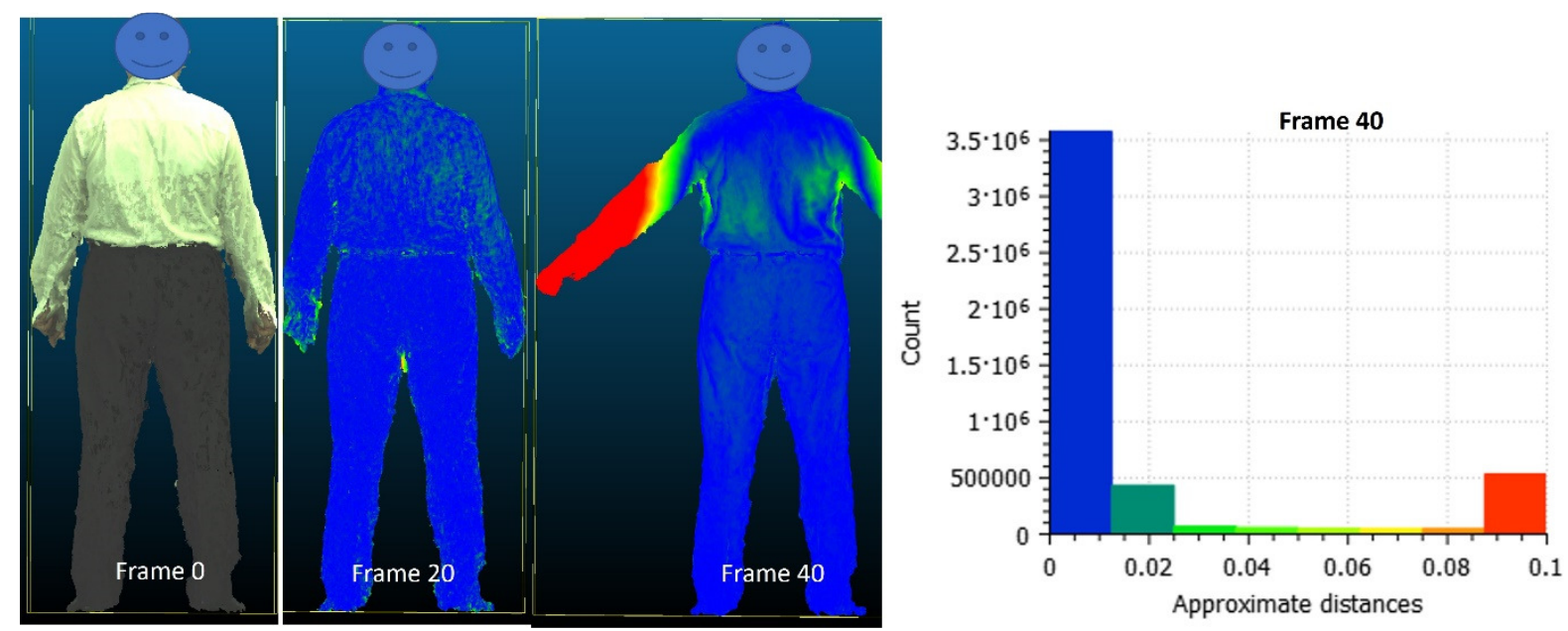

Fig. 3. Approximate distances between the frames 20, 40 to the point cloud of initial frame. Points at larger than $10 \mathrm{~cm}$ distance to the original cloud are sorted in the class with $10 \mathrm{~cm}$ and more (red color). 

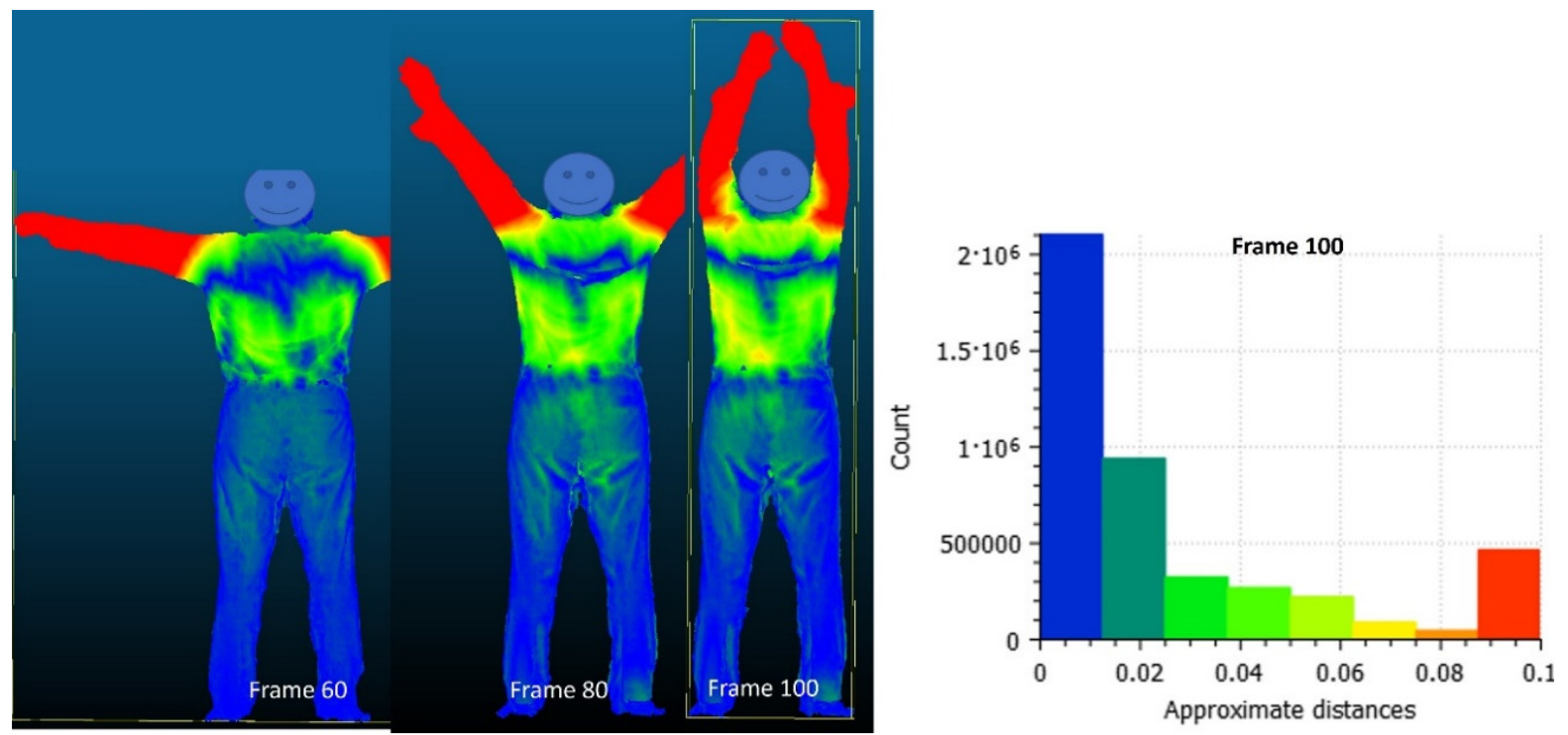

Fig. 4. Approximate distances between the frames 60,80 and 100 to the point cloud of initial frame. Points at larger than $10 \mathrm{~cm}$ distance to the original cloud are sorted in the class with $10 \mathrm{~cm}$ and more (red color).

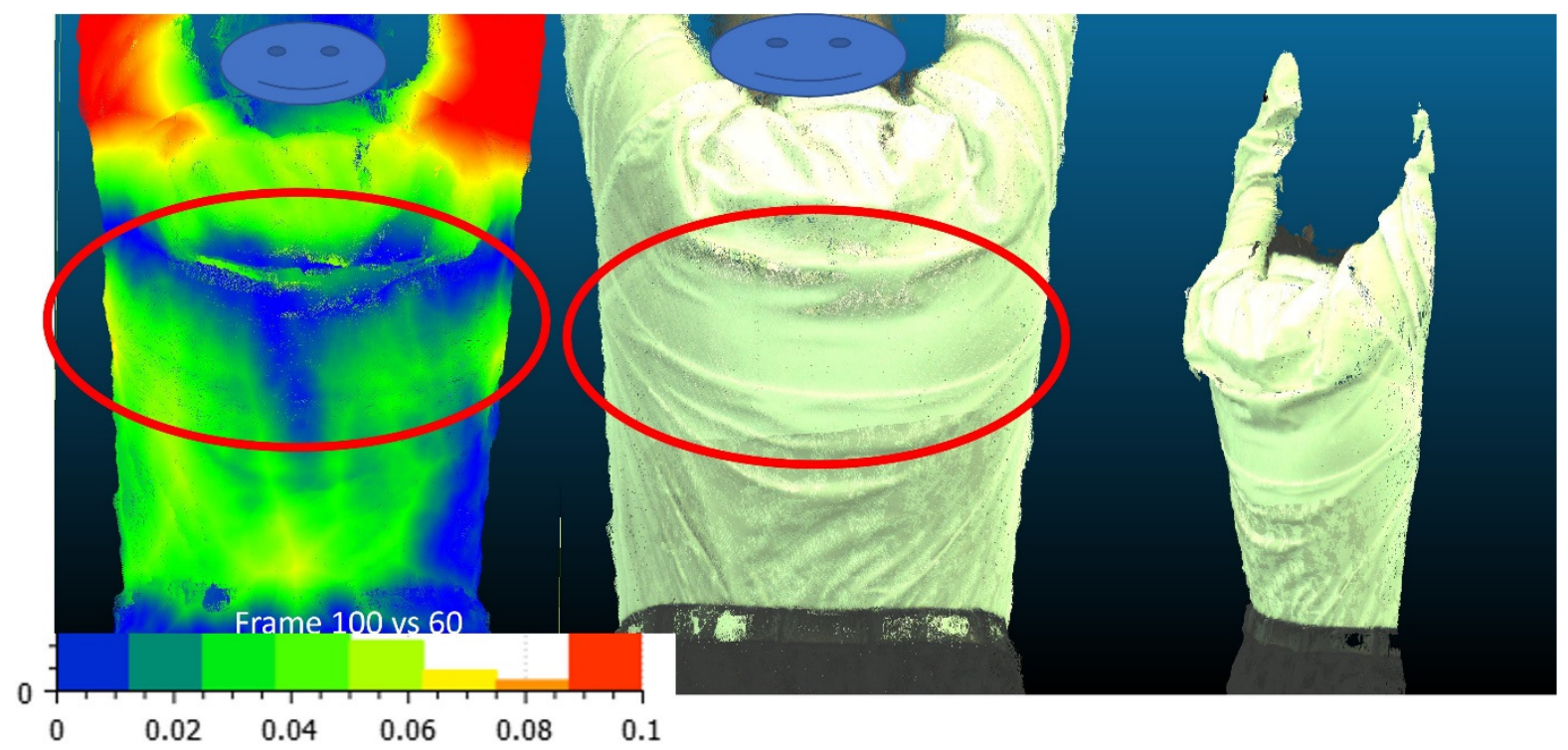

Fig. 5. Relative distance between frame 100 (hands up) and frame 60 (frames horizontally) as and the raw points from two projections of frame 100, demonstrating the sticking between the shirt and body.

Figure 3 presents the initial state frame, and frame 20. The approximate distance between these two frames with no explicit motion is as expected significantly lower than $1 \mathrm{~cm}$, but in order to keep the data comparable between the frames, the same color scale was kept for all frames. For all frames, the distances between the cloud points larger than $10 \mathrm{~cm}$ are ignored, and represented in red color as belonging to the largest class (10 $\mathrm{cm}$ and larger), as in the current case they represents the body motion parts and are not giving important information for the clothing. The comparison of the points clouds at frame 40 (Fig 3), and frames 60, 80 and 100 (Fig 4) to the initial frame (Frame 000) demonstrates that the distance between the clouds increases only in some areas and these are corresponding to the real shirt motion. The distances between the point clouds in the area of trousers do not significantly change, small motion is detected at frames 80 and 100 in the same time as the shirt is moved up. At the same time the shirt is sliding up when the sleeves are starting to move up with the hands and starts going out of the trousers. Frame 80 and 100 shows small region close to the waist with displacements larger than 5-6 cm. This corresponds to the reality. Another interesting observation is that the line around the back and the shoulder blades remains fixed on the body during this motion step. In Figure 2, frame 100 with the raw points, this sticking effect is well recognizable. The distances were checked as well between the frame 100 (hand up) and frame 60 (hands horizontally) as depicted 
in figure 5, where the distance map shows that after the hands passed the horizontal direction and continued moving up, in the areas of the waist the relative motion is running and the shoulder blades region is sticking on the body.

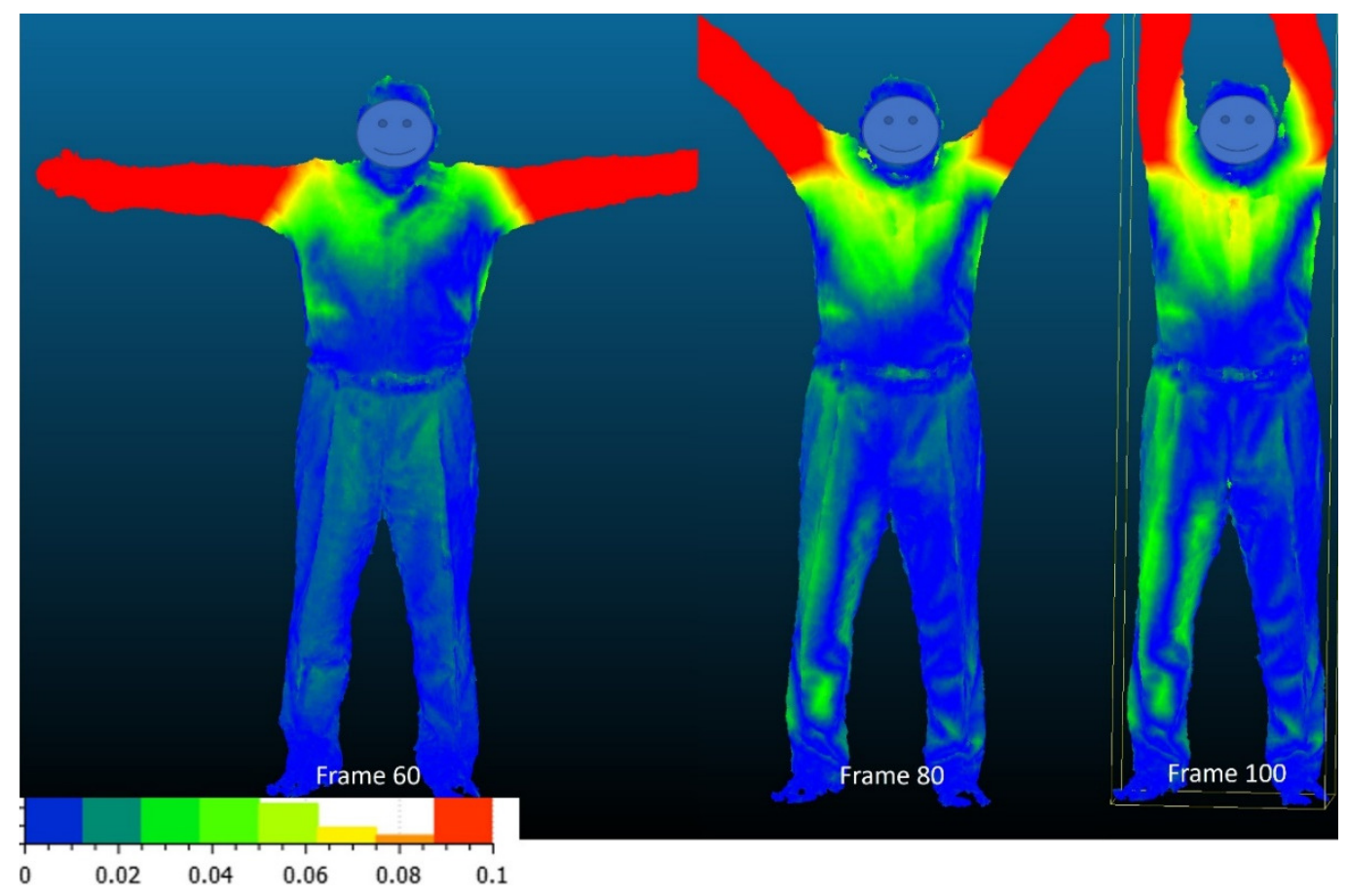

Fig. 6. Distances between the point clouds of frames 60,80 and 100 and the initial frame, visualized in the front side. Distances larger than $10 \mathrm{~cm}$ are sorted in the class 10 and larger (red color).

The analysis of the front side of the scan shows some more motion of the trousers. It marks well the areas in blue where the shirt remains fixed. This is a clear motion in the area of the chest and a recognizable motion under the sleeves. Both analysis of the point cloud distances between the current frames and the initial one for the front and back parts of the scanned human with moving hands, demonstrate very good the correspondence of the values to the real slippage of the shirt. It shows that the method can be used for quick analysis of the behavior of the clothing within scanned sequences.

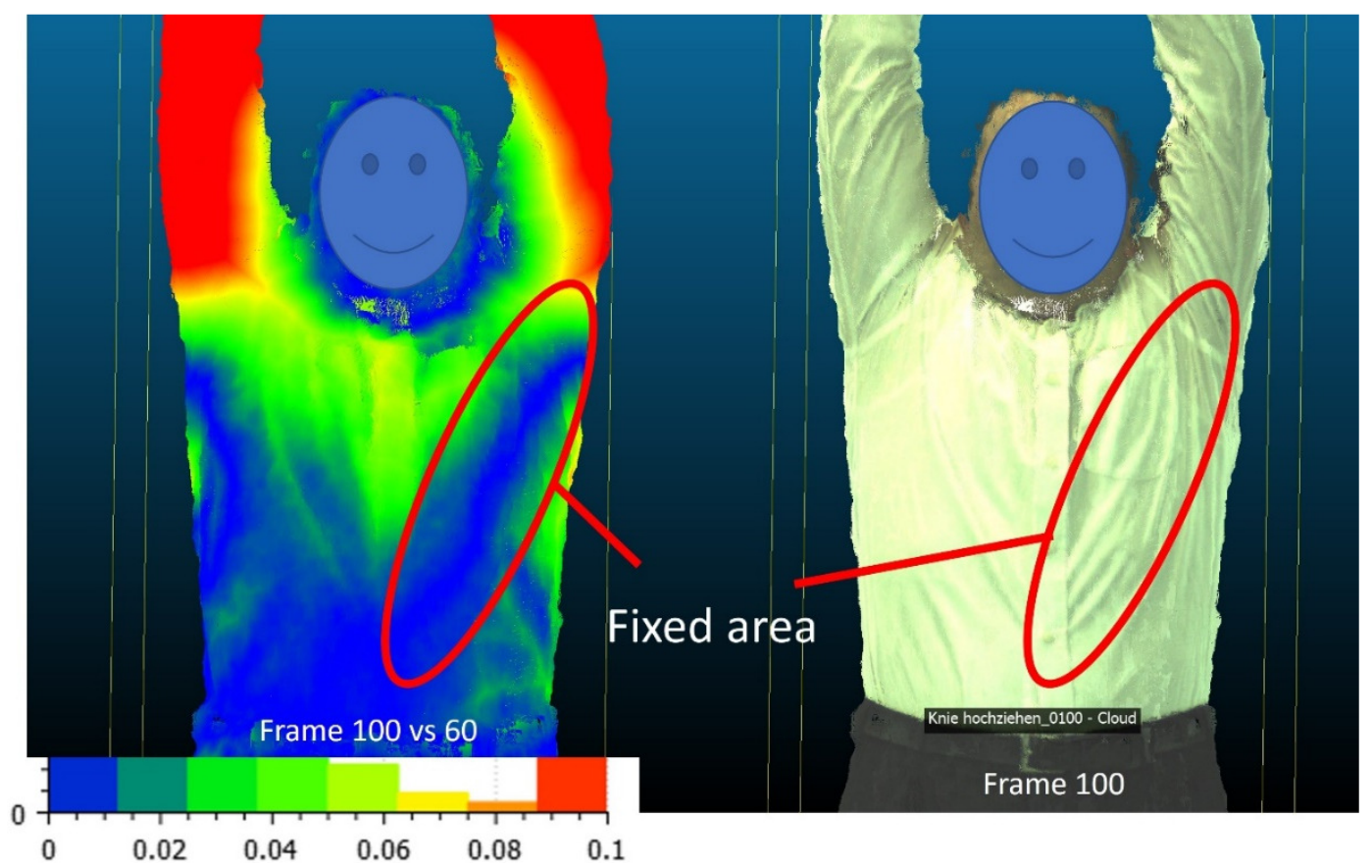

Fig. 7. Point cloud distances between the frames 100 and frame 60 in the front side of the shirt. 


\section{Conclusions}

A Human with not tight to the body clothing and moving arms from down to up was scanned with the high speed 3D scanner Move4D at a frequency of 30 frames per second. The exported triangulated mesh data provide good information for analysis of the motion of the shirt, but at the current mesh size, two effects become filtered. The small folds and the area where the shirt was sticking on the body were not able to be recognized. Despite of this, the triangulated mesh can be used as excellent basis for following detailed analysis of the clothing during the motion. The analysis of the distances between the raw point clouds demonstrated very good optical correlation between the real slippage and sticking of the shirt during the motion and the computed distances between the frames. The computation of the distances has taken less seconds and can be applied for more frames than the analyzed few in this paper. It can be used as quick method for analysis of the clothing behavior during the body motion. The combination of high speed scanning with fast analysis methods of the scanned data makes the optimization of the clothing construction for larger number of postures and motion types possible, which was not possible until now.

\section{References}

[1] IBV, MOVE4D. Spain: Instituto de Biomecánica - IBV, 2020. Accessed: 2021. [Online]. Available: https://www.ibv.org/tecnologias/analisis-de-movimientos-4d/move-4d-3/

[2] 3dMDbody System. [Online]. Available: https://3dmd.com/

[3] E. Mert, A. Psikuta, M.-A. Bueno, and R. M. Rossi, "The effect of body postures on the distribution of air gap thickness and contact area," International Journal of Biometeorology, pp. 363-375.

[4] Y. Nam, "Analysis of Clothing Air Gap in a Protective Suit According to the Body Postures," Journal of Fiber Bioengineering and Informatics, vol. 7, no. 4, pp. 573-581, 2014, doi: 10.3993/jfbi12201410.

[5] Y. Wang, "Effects of Body Postures on Clothing Air Gap in Protective Clothing," Journal of Fiber Bioengineering and Informatics, vol. 4, no. 3, pp. 277-283, 2011, doi: 10.3993/jfbi09201107.

[6] D. ZHANG, S. Krzywinski, and Y. KYOSEV, "Analysis of Clothing Deformation During Motion and its Application for the Design of Functional Clothing," in Proceedings of 3DBODY.TECH 2020 11th International Conference and Exhibition on 3D Body Scanning and Processing Technologies, Online/Virtual, 17-18 November 2020, Online, Nov. 2020 - Nov. 2020.

[7] C. Loercher, S. Morlock, and A. Schenk, "Design of a Motion-Oriented Size System for Optimizing Professional Clothing and Personal Protective Equipment," J Fashion Technol Textile Eng, s4, 2018, doi: 10.4172/2329-9568.S4-014.

[8] C. PIRCH, A. KLEPSER, and S. Morlock, "Using 3D Scanning to Create 4D Motion Data for Clothing Simulation," in Proceedings of 3DBODY.TECH 2020 - 11th International Conference and Exhibition on 3D Body Scanning and Processing Technologies, Online/Virtual, 17-18 November 2020, Online, Nov. 2020 - Nov. 2020.

[9] C. Lassner, G. Pons-Moll, and P. V. Gehler, "A Generative Model of People in Clothing," in Proceedings IEEE International Conference on Computer Vision (ICCV), 2017. [Online]. Available: http://files.is.tuebingen.mpg.de/classner/gp/

[10] G. Pons-Moll, S. Pujades, S. Hu, and M. J. Black, "ClothCap," ACM Trans. Graph., vol. 36, no. 4, pp. 1-15, 2017, doi: 10.1145/3072959.3073711.

[11] Daniel Girardeau-Montaut, CloudCompare: Daniel Girardeau-Montaut, 2020. [Online]. Available: www.cloudcompare.org

[12] J. Elseberg, D. Borrmann, and A. Nuchter, "Efficient processing of large 3D point clouds," in 2011 XXIII International Symposium on Information, Communication and Automation Technologies, Sarajevo, 2011, pp. 1-7. 